JIRSS (2021)

Vol. 20, No. 01, pp 101-121

DOI:10.52547/jirss.20.1.101

\title{
On Burr III-Inverse Weibull Distribution with COVID-19 Applications
}

\author{
Fiaz Ahmad Bhatti ${ }^{1}$, Sedigheh Mirzaei Salehabadi ${ }^{2}$, and Gholamhossein G. Hamedani ${ }^{3}$ \\ ${ }^{1}$ National College of Business Administration and Economics, Lahore, PAKISTAN. \\ 2 St. Jude Children's Research Hospital, Memphis, TN, USA. \\ ${ }^{3}$ Marquette University, Milwaukee, WI 53201-1881, USA.
}

Received: 28/06/2020, Revision received: 30/01/2021, Published online: 03/04/2021

\begin{abstract}
We introduce a flexible lifetime distribution called Burr III-Inverse Weibull (BIII-IW). The new proposed distribution has well-known sub-models. The BIII-IW density function includes exponential, left-skewed, right-skewed and symmetrical shapes. The BIII-IW model's failure rate can be monotone and non-monotone depending on the parameter values. To show the importance of the BIII-IW distribution, we establish various mathematical properties such as random number generator, ordinary moments, conditional moments, residual life functions, reliability measures and characterizations. We address the maximum likelihood estimates (MLE) for the BIII-IW parameters and estimate the precision of the maximum likelihood estimators via a simulation study. We consider applications to two COVID-19 data sets to illustrate the potential of the BIII-IW model.
\end{abstract}

Keywords. Moment, Reliability, Characterizations, Maximum Likelihood Estimation.

MSC: 62E10,62E15, 62F10, 62P10.

Corresponding Author: Fiaz Ahmad Bhatti (fiazahmad72@gmail.com)

Sedigheh Mirzaei Salehabadi (Sedigheh.Mirzaei@stjude.org)

Gholamhossein G. Hamedani (g.hamedani@mu.edu) 


\section{Introduction}

The selection of a suitable model for data analysis is generally quite challenging. If a wrong model is applied to analyze the dataset it leads to loss of information and invalid inferences. It is obligatory to identify the most suitable model for the given dataset. In the recent decade, many continuous distributions have been introduced in statistical literature. Some of these distributions, however, are not flexible enough for analysis of lifetime data. Hence, more versatile models are needed. The generalization techniques such as either inserting one or more shape parameters or transforming of the parent distribution are useful to (i) increase the applicability of a parent distribution; (ii) explore skewness and tail properties and (iii) improve the goodness-of-fit of the generalized distributions.

The inverse Weibull distribution (Keller and Kanath, 1982), developed to study the decay of mechanical components in survival and reliability analysis, is a highly flexible yet simple model. Variations and generalizations of the inverse Weibull distribution have been of great interest in literature: beta inverse Weibull (B-IW) (Khan , 2010), Kumaraswamy-Inverse Weibull (Kw-IW) (Shahbaz, et al. ,2012), reflected generalized beta inverse Weibull (Elbatal, et al. , 2016)), Topp-Leone inverse Weibull (Abbas, et al. , 2017), Odd Frechet inverse Weibull (OF-IW) (Fayomi , 2019) and gamma inverse Weibull(G-IW) (Abbas, et al. , 2020).

The main idea of the present paper is to incorporate the inverse Weibull distribution into a larger family through an application of the Burr III distribution. In fact, based on the T-X transform defined by Alzaatreh, et al. (2013), we construct the BIII-IW distribution. The new model has flexible shapes to model various lifetime data sets. Additionally, its special sub-models produce better fits than other well-known models. Here we study the BIII-IW distribution with COVID-19 applications.

Our study of the BIII-IW distribution is based on the following motivations: (i) to generate distributions with symmetrical, right-skewed, left-skewed, J, reverse-J, exponential shaped as well as high kurtosis; (ii) to have monotone and non-monotone failure rate function; (iii) to derive mathematical properties such as random number generator, ordinary moments, conditional moments, residual life functions, reliability measures and characterizations; (iv) to estimate the precision of the maximum likelihood estimators via a simulation study; (v) to reveal the potentiality and utility of the BIII-IW model; (vi) to work as the preeminent substitute model to other existing models; (vii) to deliver better fits than other models and (viii) to infer empirically from goodness of 
fit statistics (GOFs) and graphical tools.

This article is structured as follows. Section 2 derives the BIII-IW model and studies its basic structural properties, random number generator and sub-models. Section 3 presents certain mathematical properties such as ordinary moments, conditional moments, residual life functions, reliability measures and characterizations. In Section 4, we address the maximum likelihood estimation for the BIII-IW parameters. We evaluate the precision of the maximum likelihood estimators via a simulation study. In Section 5, we consider applications to two COVID-19 data sets to elucidate the potentiality of the BIII-IW model. In Section 6, we offer some concluding remarks.

\section{The BIII-IW Distribution}

We derive the BIII-IW distribution from the T-X family technique. We highlight the shapes of the density and failure rate functions.

\subsection{T-X Family Technique}

The cumulative distribution function (cdf) and probability density function (pdf) of the inverse Weibull distribution are given, respectively, by

$$
G(x ; \lambda, \eta)=\exp \left(-\lambda x^{-\eta}\right), \quad x \geq 0,
$$

and

$$
g(x ; \lambda, \eta)=\lambda \eta x^{-\eta-1} \exp \left(-\lambda x^{-\eta}\right), \quad x>0, \lambda>0, \eta>0,
$$

The odds ratio for the inverse Weibull random variable $X$ is

$$
W(G(x ; \lambda, \eta))=\frac{G(x ; \lambda, \eta)}{\bar{G}(x ; \lambda, \eta)}=\frac{e^{-\lambda x^{-\eta}}}{1-e^{-\lambda x^{-\eta}}}=\left[\exp \left(\lambda x^{-\eta}\right)-1\right]^{-1}
$$

The cdf of the T-X family (Alzaatreh, et al. ,2013) of distributions has the form

$$
F(x)=\int_{a}^{W[G(x ; \xi)]} r(t) d t, \quad x \in \mathbb{R},
$$

where $r(t)$ is the pdf of the random variable (rv) $T$, where $T \in[a, b]$ for $-\infty<a<b<\infty$ and $W[G(x ; \xi)]$ is a function of the baseline cdf of a rv $X$ with the parameter vector $\xi$, which satisfies the conditions: 
- $W[G(x ; \xi)] \in[a, b]$,

- $W[G(x ; \xi)]$ is differentiable and monotonically non-decreasing and

- $\lim _{x \rightarrow-\infty} W[G(x ; \xi)] \rightarrow a$ and $\lim _{x \rightarrow \infty} W[G(x ; \xi)] \rightarrow b$.

The pdf of the $T-X$ family can be expressed as

$$
f(x)=\left\{\frac{\partial}{\partial x} W[G(x ; \xi)]\right\} r\{W[G(x ; \xi)]\}, \quad x \in \mathbb{R} .
$$

We derive the cdf of the BIII-IW distribution from the T-X family technique by setting

$$
r(t)=\alpha \beta t^{-\beta-1}\left(1+t^{-\beta}\right)^{-\alpha-1}, \quad t>0, \alpha>0, \beta>0,
$$

and

$$
W(G(x ; \lambda, \eta))=\frac{G(x ; \lambda, \eta)}{\bar{G}(x ; \lambda, \eta)}=\frac{e^{-\lambda x^{-\eta}}}{1-e^{-\lambda x^{-\eta}}}=\left[\exp \left(\lambda x^{-\eta}\right)-1\right]^{-1} .
$$

The cdf of the BIII-IW distribution takes the form

$$
F(x)=\left[1+\left(e^{\lambda x^{-\eta}}-1\right)^{\beta}\right]^{-\alpha} \quad x \geq 0,
$$

where $\alpha, \beta, \lambda, \eta$ are the parameters. The BIII-IW density can be expressed as

$$
f(x)=\alpha \beta \lambda \eta x^{-\eta-1} e^{\lambda x^{-\eta}}\left(e^{\lambda x^{-\eta}}-1\right)^{\beta-1}\left[1+\left(e^{\lambda x^{-\eta}}-1\right)^{\beta}\right]^{-\alpha-1} \quad x>0 .
$$

Hereafter, a random variable with pdf (2.4) is denoted by $X \sim B I I I-I W(\alpha, \beta, \lambda, \eta)$. If $X \sim B I I I-I W(\alpha, \beta, \lambda, \eta)$, the survival function, failure rate, reverse failure rate and cumulative failure rate of $X$ are given, respectively, by (for $x>0$ )

$$
\begin{gathered}
S(x)=1-\left[1+\left(e^{\lambda x^{-\eta}}-1\right)^{\beta}\right]^{-\alpha}, \\
\lambda(x)=\frac{\alpha \beta \lambda \eta x^{-\eta-1} e^{\lambda x^{-\eta}}\left(e^{\lambda x^{-\eta}}-1\right)^{\beta-1}\left[1+\left(e^{\lambda x^{-\eta}}-1\right)^{\beta}\right]^{-\alpha-1}}{1-\left[1+\left(e^{\lambda x^{-\eta}}-1\right)^{\beta}\right]^{-\alpha}}, \\
r(x)=\frac{d}{d x} \ln \left[1+\left(e^{\lambda x^{-\eta}}-1\right)^{\beta}\right]^{-\alpha}
\end{gathered}
$$

and

$$
\Lambda(x)=-\ln \left\{1-\left[1+\left(e^{\lambda x^{-\eta}}-1\right)^{\beta}\right]^{-\alpha}\right\}
$$


The quantile function of $X$ (for $0<q<1$ ) follows from

$$
x_{q}=\left\{\ln \left[\left(q^{-\frac{1}{\alpha}}-1\right)^{\frac{1}{\beta}}+1\right]^{\frac{1}{\lambda}}\right\}^{-\frac{1}{\eta}},
$$

and its random number generator with $Z \sim$ Uniform $(0,1)$ is the solution of the nonlinear equation

$$
X=\left\{\ln \left[\left(Z^{-\frac{1}{\alpha}}-1\right)^{\frac{1}{\beta}}+1\right]^{\frac{1}{\lambda}}\right\}^{-\frac{1}{\eta}} .
$$

The sub-models of BIII-IW distribution are (i) For $\eta=2$, the BIII-IW distribution reduces to Burr III-inverse Rayleigh (BIII-IR); (ii) For $\eta=1$, the BIII-IW distribution reduces to Burr III-inverse exponential (BIII-IE); (iii) For $\beta=1$, the BIII-IW distribution reduces to inverse Lomax-inverse Weibull (IL-IW); (iv) For $\beta=1$ and $\eta=2$, the BIII-IW distribution reduces to inverse Lomax- inverse Rayleigh (IL-IR); (v) For $\beta=\eta=1$, the BIII-IW distribution reduces to inverse Lomax- inverse exponential (IL-IE); (vi) For $\alpha=1$, the BIII-IW distribution reduces to the log-logistic -inverse Weibull (LL-IW); (vii) For $\alpha=1$ and $\eta=2$, the BIII-IW distribution reduces to the log-logistic-inverse Rayleigh (LL-IR) and (viii) For $\alpha=\eta=1$, the BIII-IW distribution reduces to the log-logistic-inverse exponential (LL-IE) distribution.

\subsection{Shapes of the BIII-IW Density and Hazard Rate Functions}

We plot the density and failure rate functions of the BIII-IW distribution for selected parameter values. The BIII-IW density can display numerous shapes such as bimodal, symmetrical, right-skewed, left-skewed, J, reverse-J and exponential (Figure 1). The failure rate function can highlight shapes as modified bathtub, inverted bathtub, decreasing, increasing (Figure 2). Therefore, the BIII-IW distribution is quite flexible and can be applied to numerous data sets.

\subsection{Useful Expansions}

Consider the two binomial series

$$
(1+z)^{-\alpha}=\sum_{i=0}^{\infty}(-1)^{i}\left(\begin{array}{c}
\alpha+i-1 \\
i
\end{array}\right) z^{i} \quad|z|<1, \alpha>0,
$$

and

$$
(1-z)^{\alpha}=\sum_{m=0}^{\infty}(-1)^{m}\left(\begin{array}{l}
\alpha \\
m
\end{array}\right) z^{m}
$$


The pdf in (2.4) can be expressed as

$$
f(x)=\alpha \beta \lambda \eta x^{-\eta-1} \exp \left(\lambda x^{-\eta}\right)\left[\frac{\exp \left(-\lambda x^{-\eta}\right)}{1-\exp \left(-\lambda x^{-\eta}\right)}\right]^{-\beta+1} \underbrace{\left\{1+\left[\frac{1-\exp \left(-\lambda x^{-\eta}\right)}{\exp \left(-\lambda x^{-\eta}\right)}\right]^{\beta}\right\}^{-\alpha-1}}_{\mathrm{A}(\mathrm{x})}
$$

applying (2.5) to $A(x)$ in (2.7), we obtain

$$
\begin{aligned}
& f(x)=\alpha \beta \lambda \eta x^{-\eta-1} \exp \left(\lambda x^{-\eta}\right) \sum_{i=0}^{\infty}(-1)^{i}\left(\begin{array}{c}
\alpha+i \\
i
\end{array}\right)\left[\frac{1-\exp \left(-\lambda x^{-\eta}\right)}{\exp \left(-\lambda x^{-\eta}\right)}\right]^{\beta(i+1)-1}, \\
& f(x)=\alpha \beta \lambda \eta x^{-\eta-1} \exp \left(\lambda x^{-\eta}\right) \sum_{i=0}^{\infty}(-1)^{i}\left(\begin{array}{c}
\alpha+i \\
i
\end{array}\right)\left[\exp \left(-\lambda x^{-\eta}\right)\right]^{\beta(i+1)-1} \underbrace{\left[1-\exp \left(-\lambda x^{-\eta}\right)\right]^{\beta(i+1)-1}}_{\mathrm{B}(\mathrm{x})} .
\end{aligned}
$$

Now applying (2.6) to $B(x)$ in (2.8), we obtain

$$
f(x)=\lambda \eta x^{-\eta-1} \sum_{i, m=0}^{\infty} c_{i, m} \exp \left\{-\lambda[m-\beta(i+1)] x^{-\eta}\right\},
$$

which is density of exponentiated inverse Weibull distribution with scale parameter $\lambda[m-\beta(i+1)]$ and

$$
c_{i, m}=c_{i, m}(\alpha, \beta)=\alpha \beta(-1)^{i+m}\left(\begin{array}{c}
\alpha+i \\
i
\end{array}\right)\left(\begin{array}{c}
\beta(\alpha+i)-1 \\
m
\end{array}\right) .
$$

Equation (2.9) is the main result of this section. It reveals that the BIII-IW density is a linear combination of IW density. So, some of its mathematical properties can be easily determined from those of IW model. 


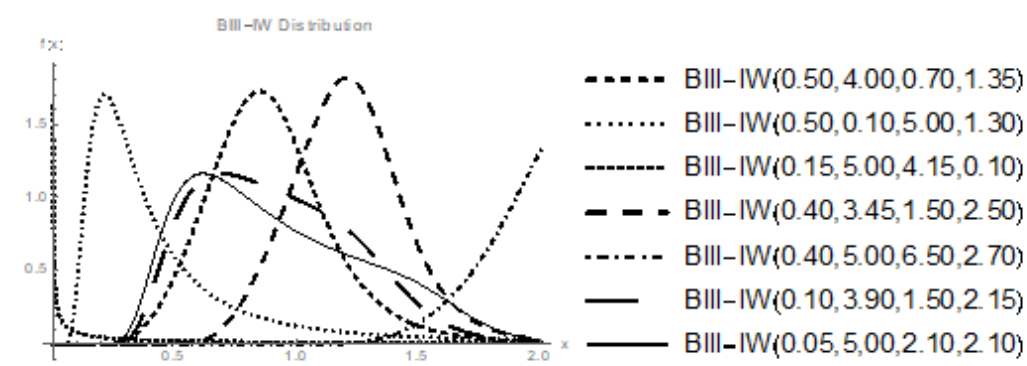

Figure 1: Plots of the BIII-IW density.

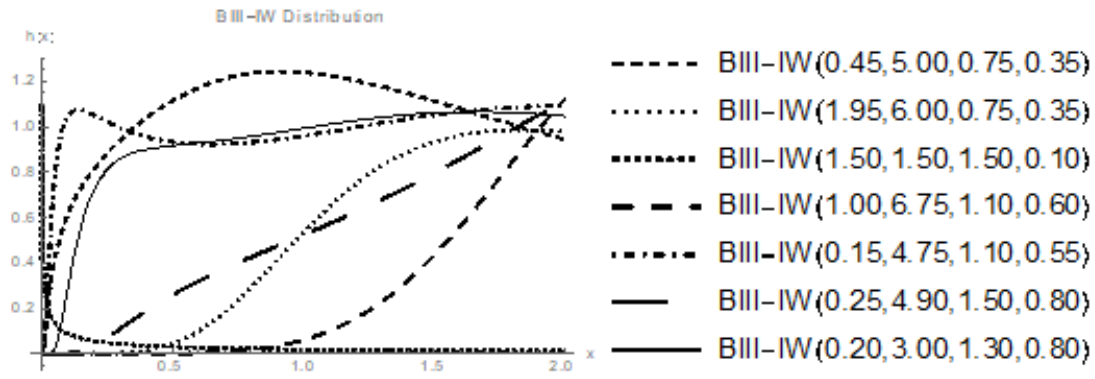

Figure 2: Plots of the BIII-IW hazard rate.

\section{Mathematical Properties}

We derive some of its mathematical properties including the ordinary moments, conditional moments, residual life functions, reliability measures and characterizations.

\subsection{Moments}

The moments are significant tools for statistical analysis in pragmatic sciences. The $\mathrm{r}^{\text {th }}$ ordinary moment of $X$ with the BIII-IW distribution is,

$$
\begin{aligned}
& E\left(X^{r}\right)=\int_{0}^{\infty} x^{r} \lambda \eta x^{-\eta-1} \sum_{i, m=0}^{\infty} c_{i, m} \exp \left\{-\lambda[m-\beta(i+1)] x^{-\eta}\right\} d x, \\
& E\left(X^{r}\right)=\lambda \eta \sum_{i, m=0}^{\infty} c_{i, m} \int_{0}^{\infty} x^{r} x^{-\eta-1} \exp \left\{-\lambda[m-\beta(i+1)] x^{-\eta}\right\} d x,
\end{aligned}
$$


Letting $\lambda[m-\beta(i+1)] x^{-\eta}=w,-\lambda \eta x^{-\eta-1} d x=\frac{d w}{[m-\beta(i+1)]}, x^{r}=\lambda^{\frac{r}{\eta}}[m-\beta(i+1)]^{\frac{r}{\eta}} w^{\frac{r}{\eta}}$, we will have

$$
\begin{aligned}
E\left(X^{r}\right) & =\lambda^{\frac{r}{\eta}} \sum_{i, m=0}^{\infty} c_{i, m}[m-\beta(i+1)]^{\frac{r}{\eta}-1} \int_{0}^{\infty} w^{1-\frac{r}{\eta}-1} e^{-w} d w \\
& =\lambda^{\frac{r}{\eta}} \sum_{i, m=0}^{\infty} c_{i, m}[m-\beta(i+1)]^{\frac{r}{\eta}-1} \Gamma\left(1-\frac{r}{\eta}\right)
\end{aligned}
$$

where $r<\eta$ and $\Gamma(\cdot)$ is the gamma function.

The $\mathrm{r}^{\text {th }}$ cenaral moment $\left(\mu_{r}\right)$, skewness $\left(\gamma_{1}\right)$ and kurtosis $\left(\gamma_{2}\right)$ for the BIII-IW model are obtained from

$$
\mu_{r}=\sum_{l=1}^{r}(-1)^{l}\left(\begin{array}{l}
r \\
l
\end{array}\right) \mu_{l}^{\prime} \mu_{r-l}^{\prime}, \quad \gamma_{1}=\frac{\mu_{3}}{\left(\mu_{2}\right)^{\frac{3}{2}}} \quad \text { and } \quad \beta_{2}=\frac{\mu_{4}}{\left(\mu_{2}\right)^{2}} .
$$

\subsection{Conditional Moments}

Life expectancy, mean waiting time and inequality measures can be obtained from the incomplete moments.

The $\mathrm{r}^{\text {th }}$ lower incomplete moment $E_{X \leq z}\left(X^{r}\right)$ is

$$
\begin{gathered}
M_{r}^{\prime}(z)=E_{X \leq z}\left(X^{r}\right)=\int_{0}^{z} x^{r} \lambda \eta x^{-\eta-1} \sum_{i, m=0}^{\infty} c_{i, m} \exp \left\{-\lambda[m-\beta(i+1)] x^{-\eta}\right\} d x, \\
E_{X \leq z}\left(X^{r}\right)=\lambda^{\frac{r}{\eta}} \sum_{i, m=0}^{\infty} c_{i, m}[m-\beta(i+1)]^{\frac{r}{\eta}-1} \int_{0}^{\infty} w^{1-\frac{r}{\eta}-1} e^{-w} d w, \\
M_{r}^{\prime}(z)=E_{X \leq z}\left(X^{r}\right)=\lambda^{\frac{r}{\eta}} \sum_{i, m=0}^{\infty} c_{i, m}[m-\beta(i+1)]^{\frac{r}{\eta}-1} \gamma\left[\left(1-\frac{r}{\eta}\right), z\right],
\end{gathered}
$$

where $\int_{0}^{w} w^{1-\frac{r}{\eta}-1} e^{-w} d w=\gamma\left[\left(1-\frac{r}{\eta}\right), w\right]$ is the lower incomplete gamma function.

The $\mathrm{r}^{\text {th }}$ conditional moment $E\left(X^{r} \mid X>z\right)$ is

$$
E\left(X^{r} \mid X>z\right)=\frac{1}{S(z)}\left\{\lambda^{\frac{r}{\eta}} \sum_{i, m=0}^{\infty} c_{i, m}[m-\beta(i+1)]^{\frac{r}{\eta}-1} \Gamma\left[\left(1-\frac{r}{\eta}\right), z\right]\right\},
$$


where $\int_{w}^{\infty} w^{1-\frac{r}{\eta}-1} e^{-w} d w=\Gamma\left[\left(1-\frac{r}{\eta}\right), w\right]$ is the upper incomplete gamma function.

The $\mathrm{r}^{\text {th }}$ conditional moment $E\left(X^{r} \mid X \leq z\right)$ is

$$
E\left(X^{r} \mid X \leq z\right)=\frac{1}{F(z)}\left\{\lambda^{\frac{r}{\eta}} \sum_{i, m=0}^{\infty} c_{i, m}[m-\beta(i+1)]^{\frac{r}{\eta}-1} \gamma\left[\left(1-\frac{r}{\eta}\right), z\right]\right\}
$$

The mean deviation about the mean $\left(\delta_{1}=E|X-\mu|\right)$ and about the median $\left(\delta_{2}=\right.$ $E|X-\tilde{\mu}|))$ can be written as $\delta_{1}=2 \mu\left(F(\mu)-M_{1}^{\prime}(\mu)\right)$ and $\delta_{2}=\mu-2 M_{1}^{\prime}(\tilde{\mu})$, respectively, where $\mu=E(X)$ and $\tilde{\mu}=x_{0.5}$. The quantities $M_{1}^{\prime}(\mu)$ and $M_{1}^{\prime}(\tilde{\mu})$ can be obtained from (3.2). For specific probability $p$, Lorenz and Bonferroni curves are computed as $L(p)=\frac{M_{1}^{\prime}(q)}{\mu}$ and $B(p)=L(p) \mid p$, where $q=Q(p)$.

\subsection{Residual Life Functions}

The $\mathrm{n}^{\text {th }}$ moments about origin of residual life, say $M_{n}(z)=E\left[(X-z)^{n} \mid X>z\right]$, of $X$ is

$$
\begin{aligned}
m_{n}(z) & =\frac{1}{S(z)} \int_{z}^{\infty}(x-z)^{r} f(x) d x \\
& =\frac{1}{S(z)} \sum_{r=0}^{n}\left(\begin{array}{l}
n \\
r
\end{array}\right)(-z)^{n-r} E_{X>z}\left(X^{r}\right) \\
& =\frac{1}{1-F(z)} \sum_{r=0}^{n}\left(\begin{array}{l}
n \\
r
\end{array}\right)(-z)^{n-r}\left\{\lambda^{\frac{r}{\eta}} \sum_{i, m=0}^{\infty} c_{i, m}[m-\beta(i+1)]^{\frac{r}{\eta}-1} \Gamma\left[\left(1-\frac{r}{\eta}\right), z\right]\right\} .
\end{aligned}
$$

The average remaining lifetime of a component at time $z$, say $m_{1}(z)$, or life expectancy called mean residual life (MRL) function is

$$
m_{1}(z)=\frac{1}{1-F(z)} \sum_{r=0}^{1}\left(\begin{array}{l}
1 \\
r
\end{array}\right)(-z)^{1-r}\left\{\lambda^{\frac{r}{\eta}} \sum_{i, m=0}^{\infty} c_{i, m}[m-\beta(i+1)]^{\frac{r}{\eta}-1} \Gamma\left[\left(1-\frac{r}{\eta}\right), z\right]\right\} .
$$

The $\mathrm{n}^{\text {th }}$ moments about origin reverse residual life, say $M_{n}(z)=E\left[(z-X)^{n} \mid X \leq z\right]$, of $X$ 
with BIII-IW distribution is

$$
\begin{aligned}
M_{n}(z) & =\frac{1}{F(z)} \int_{0}^{z}(z-x)^{r} f(x) d x \\
& =\frac{1}{F(z)} \sum_{r=0}^{n}(-1)^{r}\left(\begin{array}{l}
n \\
r
\end{array}\right)(z)^{n-r} E_{X \leq z}\left(X^{r}\right) \\
& =\frac{1}{F(z)} \sum_{r=0}^{n}(-1)^{r}\left(\begin{array}{l}
n \\
r
\end{array}\right)(z)^{n-r}\left\{\lambda^{\frac{r}{\eta}} \sum_{i, m=0}^{\infty} c_{i, m}[m-\beta(i+1)]^{\frac{r}{\eta}-1} \gamma\left[\left(1-\frac{r}{\eta}\right), z\right]\right\} .
\end{aligned}
$$

The waiting time $\mathrm{z}$ for failure of a component has passed with condition that this failure had happened in the interval $[0, z]$ is called mean waiting time (MWT) or mean inactivity time. The waiting time $z$ for failure of a component for the BIII-IW distribution is defined by

$$
M_{1}(z)=\frac{1}{F(z)} \sum_{r=0}^{1}(-1)^{r}\left(\begin{array}{l}
1 \\
r
\end{array}\right)(z)^{1-r}\left\{\lambda^{\frac{r}{\eta}} \sum_{i, m=0}^{\infty} c_{i, m}[m-\beta(i+1)]^{\frac{r}{\eta}-1} \gamma\left[\left(1-\frac{r}{\eta}\right), z\right]\right\} .
$$

\subsection{Reliability Estimation in Multi-Component Stress-Strength Model}

The multi-component stress-strength model provides about the awareness of life of a component or a system in reliability. Consider a system with $\kappa$ identical elements, out of which s elements are operative. Let $X_{i}, i=1,2 \ldots \kappa$, represent strengths of $\kappa$ elements with the $\operatorname{cdf} F$ while, the stress $Y$ enforced on the elements has the cdf $G$. The strengths $X_{i}$ and stress $Y$ are independently and identically distributed (i.i.d.). The probability that system operates properly, is the reliability of the system, i.e.

$$
\begin{aligned}
R_{s, \kappa} & =P\left[\text { strengths }\left(X_{i}, i=1,2, \ldots ., \kappa\right)>\operatorname{stress}(Y)\right] \\
& =P\left[\text { at the minimum' } s^{\prime} \text { of }\left(X_{i}, i=1,2, \ldots, \kappa\right) \text { exceed }(Y)\right] .
\end{aligned}
$$

Then, we can write this probability (Bhattacharyya and Johnson , 1974) as follows:

$$
R_{s, \kappa}=\sum_{\ell=s}^{\kappa}\left(\begin{array}{l}
\kappa \\
\ell
\end{array}\right) \int_{-\infty}^{\infty}[1-F(y)]^{\ell}[F(y)]^{\kappa-\ell} d G(y)
$$

Let $X \sim \operatorname{BIII-IW}\left(\alpha_{1}, \beta, \lambda, \eta\right), Y \sim \operatorname{BIII-IW}\left(\alpha_{2}, \beta, \lambda, \eta\right)$ with unknown $\alpha_{1}$ and $\alpha_{2}, \operatorname{common} \beta$, $\lambda, \eta$ where $X$ and $Y$ are independent. The reliability in multi-component stress-strength 
for the BIII-ME distribution is given by $R_{s, \kappa}=\sum_{\ell=s}^{\kappa}\left(\begin{array}{l}\kappa \\ \ell\end{array}\right) \int_{0}^{\infty}\left\{1-\left[1+\left(e^{\lambda x^{-\eta}}-1\right)^{\beta}\right]^{-\alpha_{1}}\right\}^{\ell}\left[1+\left(e^{\lambda x^{-\eta}}-1\right)^{\beta}\right]^{-\alpha_{1}(\kappa-\ell)} \alpha_{2} \beta \lambda \eta x^{-\eta-1}\left(e^{\lambda x^{-\eta}}-1\right)^{\beta-1}\left[1+\left(e^{\lambda x^{-\eta}}-1\right)^{\beta}\right]^{-\alpha_{2}-1} d x$. Letting $u=\left[1+\left(e^{\lambda x^{-\eta}}-1\right)^{\beta}\right]^{-\alpha_{2}}$, we obtain

$$
R_{s, \kappa}=\sum_{\ell=s}^{\kappa}\left(\begin{array}{l}
\kappa \\
\ell
\end{array}\right) \int_{0}^{1}\left(1-u^{v}\right)^{\ell} u^{v(\kappa-\ell)} d u,
$$

where $v=\frac{\alpha_{1}}{\alpha_{2}}$.

Let $w=u^{v}, u=w^{\frac{1}{v}}, d u=\frac{1}{v} w^{\frac{1}{v}-1} d w$, then we have

$$
\begin{aligned}
R_{s, \kappa} & =\sum_{\ell=s}^{\kappa}\left(\begin{array}{l}
\kappa \\
\ell
\end{array}\right) \int_{0}^{1}(1-w)^{\ell} w^{(\kappa-\ell)} \frac{1}{v} w^{\frac{1}{v}-1} d w, \\
& =\frac{1}{v} \sum_{\ell=s}^{\kappa}\left(\begin{array}{l}
\kappa \\
\ell
\end{array}\right) B\left(\ell+1, \kappa-\ell+\frac{1}{v}\right),
\end{aligned}
$$

where $B(\cdot)$ is the Beta function and $v=\frac{\alpha_{1}}{\alpha_{2}}$. The probability in (3.4) is known as the reliability in multi-component stress-strength model. For $s=k=1$, the multicomponent stress-strength model reduces to the stress-strength model (Kotz, et al. , 2003) $R_{1,1}=\frac{\alpha_{1}}{\left(\alpha_{1}+\alpha_{2}\right)}$, where $\alpha_{1}+\alpha_{2}>0$.

\subsection{Characterization via Truncated Moment}

We characterize the BIII-IW distribution via truncated moment (Glänzel , 1987).

Proposition 3.1. Let $X: \Omega \rightarrow(0, \infty)$ be a continuous random variable and let $h_{1}(x)=$ $\frac{1}{\alpha}\left[1+\left(e^{\lambda x^{-\eta}}-1\right)^{\beta}\right]^{\alpha+1}, \quad x>0$ and $h_{2}(x)=\frac{2}{\alpha}\left(e^{\lambda x^{-\eta}}-1\right)^{\beta}\left[1+\left(e^{\lambda x^{-\eta}}-1\right)^{\beta}\right]^{\alpha+1}, \quad x>0$. The random variable $X$ has the pdf (2.4) iff, the function $v(x)=\frac{E\left[h_{1}(X) \mid X \geq x\right]}{E\left[h_{2}(X) \mid X \geq x\right]}$ has the form $v(x)=\left(e^{\lambda x^{-\eta}}-1\right)^{\beta}, x>0$.

Proof. if $X$ has the pdf (2.4), then

$$
\begin{aligned}
& (1-F(x)) E\left(h_{1}(x) \mid X \geq x\right)=\left(e^{\lambda x^{-\eta}}-1\right)^{\beta}, x>0, \\
& (1-F(x)) E\left(h_{2}(x) \mid X \geq x\right)=\left(e^{\lambda x^{-\eta}}-1\right)^{2 \beta}, x>0, \\
& \frac{E\left[h_{1}(X) \mid X \geq x\right]}{E\left[h_{2}(X) \mid X \geq x\right]}=v(x)=\left(e^{\lambda x^{-\eta}}-1\right)^{-\beta}, x>0 .
\end{aligned}
$$


Conversely, if $v(x)$ has the given form, then $v^{\prime}(x)=\beta \lambda \eta x^{-\eta-1} e^{\lambda x^{-\eta}}\left(e^{\lambda x^{-\eta}}-1\right)^{-\beta-1}, x>0$. The differential equation

$$
s^{\prime}(x)=\frac{v^{\prime}(x) h_{2}(x)}{v(x) h_{2}(x)-h_{1}(x)}=\frac{2 \beta \lambda \eta x^{-\eta}-1}{e^{\lambda x^{-\eta}}},
$$

has soltion $s(x)=\ln \left(e^{\lambda x^{-\eta}}-1\right)^{-2 \beta}, x>0$. Therefore, in light of Theorem G (Glänzel , 1987), $X$ has the pdf (2.4).

Corollary 3.1. Let $X: \Omega \rightarrow(0, \infty)$ be a continuous random variable and let $h_{2}(x)=\frac{2}{\alpha}\left(e^{\lambda x^{-\eta}}-\right.$ $1)^{\beta}\left[1+\left(e^{\lambda x^{-\eta}}-1\right)^{\beta}\right]^{\alpha+1}, \quad x>0$. The $p d f$ of $X$ is (2.4) iff there exist function $v(x)$ and $h_{1}(x)$ satisfying the differential equation

$$
\frac{v^{\prime}(x) h_{2}(x)}{v(x) h_{2}(x)-h_{1}(x)}=\alpha \beta \lambda \eta x^{-\eta-1} e^{\lambda x^{-\eta}}\left(e^{\lambda x^{-\eta}}-1\right)^{-\beta-1}\left[1+\left(e^{\lambda x^{-\eta}}-1\right)^{\beta}\right]^{-\alpha-1} .
$$

Remark 1. The general solution of (3.5) is given by

$$
v(x)=\left(e^{\lambda x^{-\eta}}-1\right)^{-2 \beta}\left\{\int\left[-\alpha \beta \lambda \eta x^{-\eta-1} e^{\lambda x^{-\eta}}\left(e^{\lambda x^{-\eta}}-1\right)^{\beta-1}\left[1+\left(e^{\lambda x^{-\eta}}-1\right)^{\beta}\right]^{-\alpha-1} h_{1}(x)\right] d x+D\right\}
$$

where $D$ is a constant.

\section{Statistical Inference}

First, we adopt the maximum likelihood estimation technique for the BIII-IW parameters. We evaluate the behavior of the maximum likelihood estimators of the BIII-IW parameters via a simulation study. We explain the utility of the BIII-IW model among its family and class using real data set.

\subsection{Parameter Estimation}

Let $\xi=(\alpha, \beta, \lambda, \eta)^{T}$ be the unknown parameter vector. The log-likelihood function $\ell(\xi)$ for the BIII-IW distribution is

$$
\begin{aligned}
\ell=\ln L(\xi)= & n \ln (\alpha)+n \ln (\beta)+n \ln (\lambda)+n \ln (\eta)-(\eta+1) \sum_{i=1}^{n} \ln x_{i}+\lambda \sum_{i=1}^{n} \ln x_{i}^{-\eta} \\
& +(\beta-1) \sum_{i=1}^{n} \ln \left(e^{\lambda x_{i}^{-\eta}}-1\right)-(\alpha+1) \sum_{i=1}^{n} \ln \left[1+\left(e^{\lambda x_{i}^{-\eta}}-1\right)^{\beta}\right] .
\end{aligned}
$$


The first derivatives of $\ell(\xi)$ with respect to $\alpha, \beta, \lambda$ and $\eta$ are as given below.

$$
\begin{gathered}
\frac{\partial \ell}{\partial \alpha}=\frac{n}{\alpha}-\sum_{i=1}^{n} \ln \left[1+\left(e^{\lambda x_{i}^{-\eta}}-1\right)^{\beta}\right], \\
\frac{\partial \ell}{\partial \beta}=\frac{n}{\beta}+\sum_{i=1}^{n} \ln \left(e^{\lambda x_{i}^{-\eta}}-1\right)+(\alpha+1) \sum_{i=1}^{n}\left[1+\left(e^{\lambda x_{i}^{-\eta}}-1\right)^{-\beta}\right]^{-1} \ln \left(e^{\lambda x_{i}^{-\eta}}-1\right), \\
\frac{\partial \ell}{\partial \lambda}=\frac{n}{\lambda}+\sum_{i=1}^{n} x_{i}^{-\eta}-(\beta+1) \sum_{i=1}^{n} \frac{x_{i}^{-\eta}}{1-e^{-\lambda x_{i}^{-\eta}}}+(\alpha+1) \beta \sum_{i=1}^{n}\left[1+\left(e^{\lambda x_{i}^{-\eta}}-1\right)^{\beta}\right]^{-1}\left(e^{\left.\lambda x_{i}^{-\eta}-1\right)^{\beta-1}} x_{i}^{-\eta} e^{\lambda x_{i}^{-\eta}},\right. \\
\frac{\partial \ell}{\partial \eta}=\frac{n}{\eta}-\sum_{i=1}^{n} \ln x_{i}-\lambda \sum_{i=1}^{n} x_{i}^{-\eta} \ln x_{i}+(\beta+1) \lambda \sum_{i=1}^{n} \frac{x_{i}^{-\eta} \ln x_{i}}{\left(1-e^{-\lambda x_{i}^{-\eta}}\right)}+(\alpha+1) \beta \sum_{i=1}^{n} \frac{\left(e^{\lambda x_{i}^{-\eta}}-1\right)^{\beta-1} x_{i}^{-\eta} e^{\lambda x_{i}^{-\eta}} \ln x_{i}}{\left[1+\left(e^{\lambda x_{i}^{-\eta}}-1\right)^{\beta}\right]} .
\end{gathered}
$$

One can compute the maximum likelihood estimators (MLEs) of $\xi=(\alpha, \beta, \lambda, \eta)^{T}$ by solving the following equations $\frac{\partial \ell}{\partial \alpha}=0, \frac{\partial \ell}{\partial \beta}=0, \frac{\partial \ell}{\partial \lambda}=0$ and $\frac{\partial \ell}{\partial \eta}=0$ simultaneously, either directly or using quasi-Newton procedure, computer packages/softwares such as R, SAS, Ox, MATHEMATICA, MATLAB and MAPLE.

\section{Simulation Study}

We evaluate the behavior of the MLEs of the BIII-IW parameters regarding the sample size $n$. We generate 10000 samples of sizes $n=30,50,100,200,300,500$ from the inverse cdf of the BIII-IW distribution with true parameter settings $(\alpha, \beta, \lambda, \eta)=(0.1$, $1.25,0.75,0.1),(0.25,1.5,1.1,0.25),(0.5,1.75,1.50,0.5)$ and $(0.75,2.0,1.75,0.75)$. We estimate the MLEs $(\hat{\alpha}, \hat{\beta}, \hat{\lambda}, \hat{\eta})$ for 10000 samples from the non-linear optimization techniques. We also compute the means, biases and mean squared errors (MSE) of the MLEs. We infer from the simulation results (Table 1 ) that as the sample size $n$ increases, the means approach to the true parameter value, the estimated MSE decrease, and estimated biases drop to zero. We observe that as the shape parameter increases, MSE of estimated parameters increases. Finally, we infer that the MLEs for the BIII-IW distribution are consistent. 
Table 1: Mean, Bias and MSE of BIII-IW distribution

\begin{tabular}{|c|c|c|c|c|c|c|c|c|c|}
\hline Sample & Statistics & $\alpha=0.1$ & $\beta=1.25$ & $\lambda=0.75$ & $\eta=0.1$ & $\alpha=0.25$ & $\beta=1.5$ & $\lambda=1.1$ & $\eta=0.25$ \\
\hline \multirow{3}{*}{30} & Mean & 0.1616 & 3.2743 & 1.1538 & 0.0778 & 0.2702 & 4.9168 & 1.8524 & 0.2966 \\
\hline & Bias & 0.0616 & 2.0243 & 0.4038 & -0.0222 & 0.0202 & 3.4168 & 0.7524 & 0.0466 \\
\hline & MSE & 0.0549 & 22.3957 & 1.0981 & 8 E-04 & 0.1218 & 72.8266 & 3.6263 & 0.0135 \\
\hline \multirow{3}{*}{50} & Mean & 0.1649 & 2.2468 & 1.1830 & 0.0758 & 0.2846 & 2.9445 & 1.7614 & 0.2799 \\
\hline & Bias & 0.0649 & 0.9968 & 0.4330 & -0.0242 & 0.0346 & 1.4445 & 0.6614 & 0.0299 \\
\hline & MSE & 0.0505 & 6.7098 & 1.1651 & 8 E-04 & 0.1134 & 14.880 & 3.3008 & 0.0063 \\
\hline \multirow{3}{*}{200} & Mean & 1.4220 & 1.0979 & 0.0745 & 0.2893 & 0.2893 & 1.7197 & 1.3438 & 0.2716 \\
\hline & Bias & 0.0398 & 0.1720 & 0.3479 & -0.0255 & 0.0393 & 0.2197 & 0.2438 & 0.0216 \\
\hline & MSE & 0.0197 & 0.2796 & 0.6033 & 7 E-04 & 0.0643 & 0.3111 & 1.0288 & 0.0017 \\
\hline \multirow{3}{*}{300} & Mean & 0.1319 & 1.3633 & 1.0789 & 0.0746 & 0.2821 & 1.6322 & 1.2172 & 0.2723 \\
\hline & Bias & 0.0319 & 0.1133 & 0.3289 & -0.0254 & 0.0321 & 0.1322 & 0.1172 & 0.0223 \\
\hline & MSE & 0.0126 & 0.1452 & 0.5064 & 7 E-04 & 0.0442 & 0.1184 & 0.5211 & 0.0013 \\
\hline \multirow{3}{*}{500} & Mean & 0.1273 & 1.3146 & 1.0364 & 0.0746 & 0.2695 & 1.5732 & 1.1383 & 0.2733 \\
\hline & Bias & 0.0273 & 0.0646 & 0.2864 & -0.0254 & 0.0195 & 0.0732 & 0.0383 & 0.0233 \\
\hline & MSE & 0.0087 & 0.0611 & 0.3623 & 7 E-04 & 0.0245 & 0.0434 & 0.2682 & 0.0010 \\
\hline Sample & Statistics & $\alpha=0.5$ & $\beta=1.75$ & $\lambda=1.5$ & $\eta=0.5$ & $\alpha=0.75$ & $\beta=2.0$ & $\lambda=1.75$ & $\eta=0.75$ \\
\hline \multirow{3}{*}{30} & Mean & 0.4792 & 4.1390 & 2.2859 & 0.9614 & 1.0345 & 3.4565 & 2.0767 & 2.341 \\
\hline & Bias & -0.0208 & 2.3890 & 0.7859 & 0.4614 & 0.2845 & 1.4565 & 0.3267 & 1.591 \\
\hline & MSE & 0.3300 & 58.812 & 7.0945 & 0.6397 & 4.8049 & 33.970 & 5.5384 & 8.557 \\
\hline \multirow{3}{*}{50} & Mean & 0.4777 & 2.8230 & 2.0593 & 0.8383 & 0.9115 & 2.6572 & 1.9040 & 1.8318 \\
\hline & Bias & -0.0223 & 1.0730 & 0.5593 & 0.3383 & 0.1615 & 0.6572 & 0.1540 & 1.0818 \\
\hline & MSE & 0.2705 & 11.248 & 4.7741 & 0.2486 & 2.6098 & 7.0496 & 3.5698 & 3.3994 \\
\hline \multirow{3}{*}{100} & Mean & 0.5114 & 2.2001 & 1.6104 & 0.7673 & 0.7628 & 2.3556 & 1.6278 & 1.5034 \\
\hline & Bias & 0.0114 & 0.4501 & 0.1104 & 0.2673 & 0.0128 & 0.3556 & -0.1222 & 0.7534 \\
\hline & MSE & 0.1799 & 1.4944 & 1.9890 & 0.1212 & 0.7491 & 1.7569 & 1.7727 & 1.0839 \\
\hline \multirow{3}{*}{200} & Mean & 0.5250 & 1.9752 & 1.3225 & 0.7492 & 0.7366 & 2.2318 & 1.3774 & 1.3909 \\
\hline & Bias & 0.0250 & 0.2252 & -0.1775 & 0.2492 & -0.0134 & 0.2318 & -0.3726 & 0.6409 \\
\hline & MSE & 0.1212 & 0.4107 & 0.8442 & 0.0909 & 0.2205 & 0.8524 & 0.8580 & 0.608 \\
\hline \multirow{3}{*}{300} & Mean & 0.5204 & 1.8994 & 1.2061 & 0.7468 & 0.7460 & 2.1856 & 1.2524 & 1.3557 \\
\hline & Bias & 0.0204 & 0.1494 & -0.2939 & 0.2468 & -0.0040 & 0.1856 & -0.4976 & 0.6057 \\
\hline & MSE & 0.0867 & 0.2259 & 0.4776 & 0.0822 & 0.1486 & 0.5962 & 0.6426 & 0.5145 \\
\hline \multirow{3}{*}{500} & Mean & 0.5170 & 1.8369 & 1.1116 & 0.7468 & 0.7637 & 2.1559 & 1.1407 & 1.3246 \\
\hline & Bias & 0.0170 & 0.0869 & -0.3884 & 0.2468 & 0.0137 & 0.1559 & -0.6093 & 0.5746 \\
\hline & MSE & 0.0537 & 0.1071 & 0.3046 & 0.0744 & 0.1061 & 0.4183 & 0.5442 & 0.4419 \\
\hline
\end{tabular}




\section{COVID-19 Applications}

We consider two applications with real datasets to assess the interest in the BIII-IW model. The considered data, called the COVID-19 datasets, are presented below. Severe Acute Respiratory Syndrome Coronavirus 2 (SARS-CoV-2) is the strain of Coronavirus that causes respiratory illness known as coronavirus disease 2019 (COVID-19). COVID19 is recalled as the 'the flu of 2020'. SARS-CoV-2 is a positive-sense single stranded RNA virus (contagious in humans). Here, in the first data set, the daily new COVID-19 confirmed cases in Pakistan from 21 March to 29 May 2020 (inclusive) are analyzed (Bantan, et al. ,2020). The second data set presents the analysis of the daily new deaths due to COVID-19 in China from 23 January to 28 March 2020 (Eliwa, et al. , 2020). Table 2 reports some descriptive measures for two data sets.

Table 2: Descriptive Statistics of two datasets

\begin{tabular}{lcccccccc}
\hline Data Sets & $n$ & Min & Max & Mean & Median & $\begin{array}{c}\text { Standard } \\
\text { deviation }\end{array}$ & Skewness & Kurtosis \\
\hline Daily New Confirmed cases & 70 & 89 & 2636 & 941.7571 & 767 & 743.526 & 0.6172 & 2.182 \\
$\begin{array}{l}\text { Daily New Deaths } \\
66\end{array}$ & 3 & 150 & 49.7424 & 33 & 43.8730 & 0.8365 & 2.450 \\
\hline
\end{tabular}

We compare the BIII-IW distribution with models such as BIII-IR, Burr XII inverse Weibull (BXII-IW), Burr XII inverse Rayleigh (BXII-IR) (Hafida and Haitham , 2019), odd Frechet inverse Weibull (OF-IW) (Fayomi , 2019), Kumaraswamy-Inverse Weibull (Kw-IW) (Shahbaz, et al. , 2012), W-IW, Weibull and inverse Weibull. For selection of the optimum distribution, we compute the estimate of goodness of statistics such as Cramer-von Mises $\left.W^{*}\right)$, Anderson Darling $\left(A^{*}\right)$ and Kolmogorov-Smirnov (K-S) statistic with $p$-values and various model selection criteria such as the estimate of likelihood ratio statistic $(-2 \hat{\ell})$, Akaike information criterion (AIC), corrected Akaike information criterion (CAIC) and Bayesian information criterion (BIC) for all competing and sub distributions. We compute the MLEs for the parameters and their standard errors (SEs) (in parentheses).

\subsection{Daily New COVID-19 Confirmed Cases in Pakistan}

The Total Time on Test (TTT) plot for daily new confirmed cases is first convex and then concave (Figure 3 [left]) which infers shaped modified tub shaped hazard rate. The boxplot for daily new confirmed cases is positively skewed (Figure 3 [right]). So, 
the BIII-IW distribution is suitable to model daily new confirmed cases data.

Table 3 reports the MLEs (SE) and measures $W^{*}, A^{*}, \mathrm{~K}-\mathrm{S}$ ( $p$-values). Table 4 displays $-2 \hat{\ell}, \mathrm{AIC}, \mathrm{CAIC}$ and BIC.

From the Tables 3 and 4, it is clear that our proposed model is best fitted, with the smallest values for all statistics and maximum $p$-value.
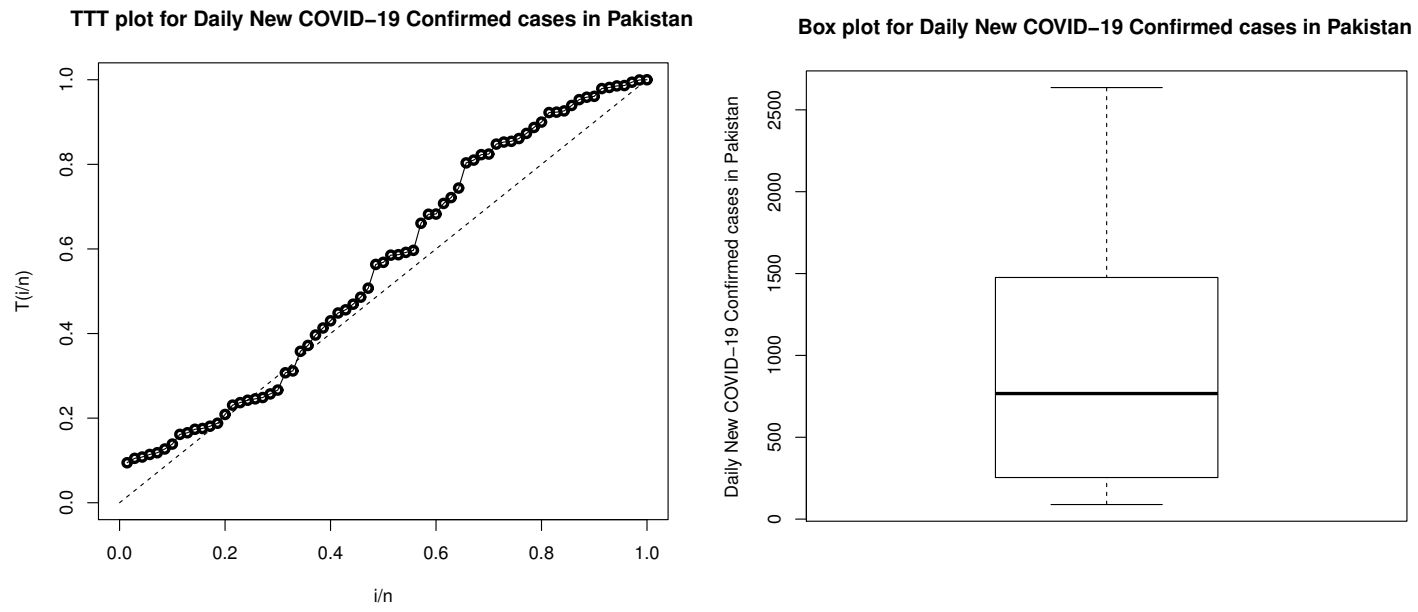

Figure 3: The Total Time on Test (TTT) plot (left), Boxplot for daily new COVID-19 confirmed cases in Pakistan (right).

Table 3: MLE (SE) and $W^{*}, A^{*}, \mathrm{~K}-\mathrm{S}$ ( $p$-values) for daily new COVID-19 confirmed cases in Pakistan

\begin{tabular}{lccccccc}
\hline Model & $\alpha$ & $\beta$ & $\lambda$ & $\eta$ & $W^{*}$ & $A^{*}$ & K-S $p$-value \\
\hline BIII-IW & $0.0091(0.0061)$ & $45.5689(33.4263)$ & $221.63(103.29)$ & $0.7314(0.0602)$ & 0.0496 & 0.3390 & $0.0738(0.8139)$ \\
BIII-IR & $92.528(517.01)$ & $0.5135(0.0473)$ & $20.926(234.19)$ & - & 0.3859 & 2.3401 & $0.1376(0.1286)$ \\
BXII-IW & $32.155(51.379)$ & $2.5230(2.6114)$ & $7.7965(12.2749)$ & $0.2313(0.1453)$ & 0.1726 & 1.1537 & $0.1151(0.2895)$ \\
BXII-IR & $0.0030(0.0001)$ & $78.493(18.875)$ & $5416.41(128.3)$ & - & 0.5300 & 3.1422 & $0.2239(0.0015)$ \\
KIW & $89.1834(54.506)$ & $0.2853(0.1560)$ & $183.66(2.5263)$ & $3.5573(1.7404)$ & 0.3892 & 2.3574 & $0.1455(0.0931)$ \\
WIW & $0.0034(0.0009)$ & $0.6860(0.2698)$ & $5.6749(3.7754)$ & $1.4694(0.4970)$ & 0.1604 & 1.0863 & $0.1153(0.2871)$ \\
OF-IW & - & $0.0680(0.0377)$ & $10.840(6.0189)$ & $1.0360(0.2307)$ & 0.4003 & 2.4195 & $0.1404(0.1147)$ \\
BIII & $426.272(218.155)$ & $1.0224(0.0914)$ & - & - & 0.3875 & 2.3487 & $0.138(0.1265)$ \\
IW & $423.106(218.146)$ & $1.0215(0.0919)$ & - & - & 0.3880 & 2.351 & $0.1382(0.1252)$ \\
\hline
\end{tabular}


Table 4: $-2 \hat{\ell}$, AIC, CAIC, BIC and HQIC for daily new COVID-19 confirmed cases in Pakistan

\begin{tabular}{lccccc}
\hline Model & $-2 \hat{\ell}$ & AIC & CAIC & BIC & HQIC \\
\hline BIII-IW & $\mathbf{1 0 7 7 . 6 5 2}$ & $\mathbf{1 0 8 5 . 6 5 2}$ & $\mathbf{1 0 8 6 . 2 6 7}$ & $\mathbf{1 0 9 4 . 6 4 6}$ & $\mathbf{1 0 8 9 . 2 2 4}$ \\
BIII-IR & 1111.445 & 1117.445 & 1117.809 & 1124.191 & 1120.125 \\
BXIII-IW & 1095.391 & 1103.391 & 1104.006 & 1112.385 & 1106.963 \\
BXII-IR & 1140.84 & 1146.84 & 1147.204 & 1153.585 & 1149.519 \\
KIW & 1114.757 & 1122.757 & 1123.372 & 1131.751 & 1126.329 \\
WIW & 1098.409 & 1106.409 & 1107.025 & 1115.403 & 1109.982 \\
OF-IW & 1112.548 & 1118.548 & 1118.912 & 1125.293 & 1121.227 \\
BIII & 1111.525 & 1115.525 & 1115.704 & 1120.022 & 1117.311 \\
IW & 1111.547 & 1115.547 & 1115.726 & 1120.044 & 1117.334 \\
\hline
\end{tabular}

Figure 4 infers that the proposed model is closely fitted to daily new COVID-19 confirmed cases.
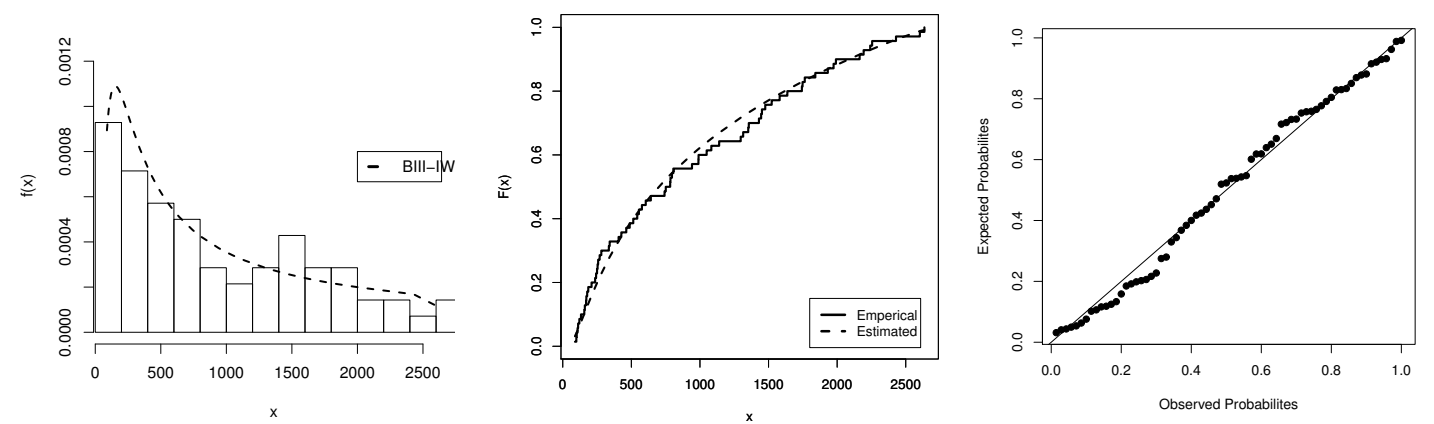

Figure 4: Fitted pdf (left), cdf (center) and P-P(right) plots of the BIII-IW distribution for daily new COVID-19 confirmed cases in Pakistan

\subsection{Daily New COVID-19 Deaths in China}

The TTT plot for daily new deaths data is first convex and then concave (Figure 5 [left]) which infers modified tub shaped hazard rate.

The boxplot for daily new deaths due to COVID-19 data is positively skewed 
(Figure 5 [right]). So, the BIII-IW distribution is suitable to model these data.

Figure 6 infers that the proposed model is closely fitted to daily new deaths. Table 5 reports the MLEs (SE) and measures $W^{*}, A^{*}, \mathrm{~K}-\mathrm{S}$ ( $p$-values).Table 6 displays $-2 \hat{\ell}, \mathrm{AIC}$, CAIC and BIC.

From the Tables 5 and 6, it is clear that our proposed model is best fitted, with the smallest values for all statistics and maximum $p$-value.
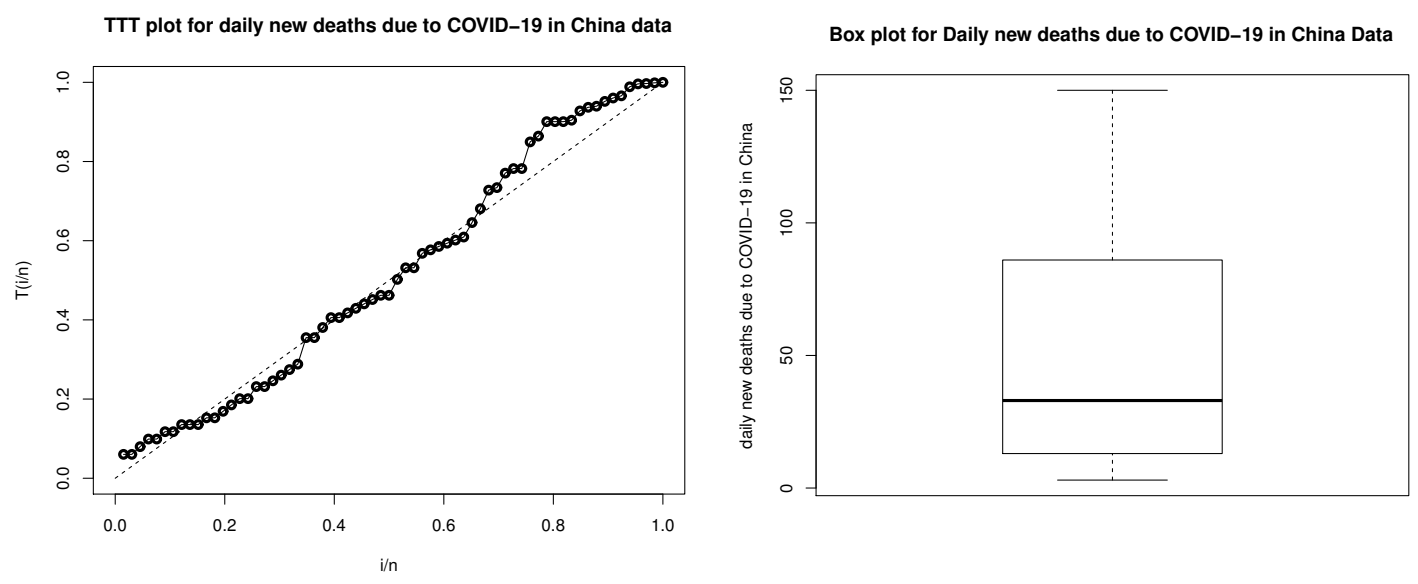

Figure 5: The Total Time on Test (TTT) plot (left), Boxplot for daily new deaths due to COVID-19 in China (right).

Table 5: MLE (SE) and $W^{*}, A^{*}, \mathrm{~K}-\mathrm{S}$ ( $p$-values) for daily new COVID-19 confirmed cases in Pakistan

\begin{tabular}{|c|c|c|c|c|c|c|c|}
\hline Model & $\alpha$ & $\beta$ & $\lambda$ & $\eta$ & $W^{*}$ & $A^{*}$ & K-S $p$-value \\
\hline BIII-IW & $0.0057(0.0023)$ & $67.6042(33.9034)$ & $21.8514(9.4536)$ & $0.6870(0.0858)$ & 0.0239 & 0.1749 & $0.0683(0.9176)$ \\
\hline BIII-IR & $5.0489(2.8062)$ & $0.4807(0.0403)$ & $12.6634(14.2521)$ & - & 0.2592 & 1.6224 & $0.1276(0.2324)$ \\
\hline BXII-IW & $84.976(250.66)$ & $2.9006(2.9932)$ & $3.3697(3.7244)$ & $0.1722(0.1074)$ & 0.0905 & 0.7054 & $0.0914(0.6402)$ \\
\hline BXII-IR & $0.4935(0.1376)$ & $0.6869(0.1438)$ & $69.5780(23.8442)$ & - & 0.3968 & 2.4200 & $0.196(0.0126)$ \\
\hline KIW & 84.9999 (47.6766) & $0.5911(0.8534)$ & $0.0406(0.1846)$ & $1.5365(2.2344)$ & 0.2859 & 1.7669 & $0.1348(0.1814)$ \\
\hline WIW & $0.1212(0.0675)$ & $0.3714(0.1894)$ & $139.90(286.04)$ & 2.7208 (1.4777) & 0.0547 & 0.4410 & $0.0876(0.6914)$ \\
\hline OF-IW & - & $25.4543(22.2713)$ & $0.7462(0.0480)$ & $0.0260(0.0226)$ & 0.2900 & 1.7893 & $0.1298(0.2159)$ \\
\hline BIII & $15.6815(3.4122)$ & $0.9489(0.0810)$ & - & - & 0.2695 & 1.6797 & $0.1258(0.247)$ \\
\hline IW & $13.5316(3.1093)$ & $0.9159(0.0837)$ & - & - & 0.2843 & 1.7583 & $0.1284(0.2263)$ \\
\hline
\end{tabular}


Table 6: $-2 \hat{\ell}$, AIC, CAIC, BIC and HQIC for daily new COVID-19 confirmed cases

\begin{tabular}{lccccc}
\hline Model & $-2 \hat{\ell}$ & AIC & CAIC & BIC & HQIC \\
\hline BIII-IW & $\mathbf{6 2 9 . 9 2 0 0}$ & $\mathbf{6 3 7 . 9 2 0 1}$ & $\mathbf{6 3 8 . 5 7 5 8}$ & $\mathbf{6 4 6 . 6 7 8 7}$ & $\mathbf{6 4 1 . 3 8 1}$ \\
BIII-IR & 660.2998 & 666.2997 & 666.6868 & 672.8687 & 668.8954 \\
BXIII-IW & 646.6522 & 654.6523 & 655.308 & 663.4109 & 658.1132 \\
BXII-IR & 673.3036 & 679.3036 & 679.6906 & 685.8725 & 681.8993 \\
KIW & 665.4548 & 673.4548 & 674.1106 & 682.2134 & 676.9158 \\
WIW & 640.0214 & 648.0214 & 648.6771 & 656.7800 & 651.4823 \\
OF-IW & 662.657 & 668.6569 & 669.044 & 675.2259 & 671.2526 \\
BIII & 661.3306 & 665.3306 & 665.5211 & 669.7099 & 667.0611 \\
IW & 662.2032 & 666.2032 & 666.3936 & 670.5825 & 667.9336 \\
\hline
\end{tabular}
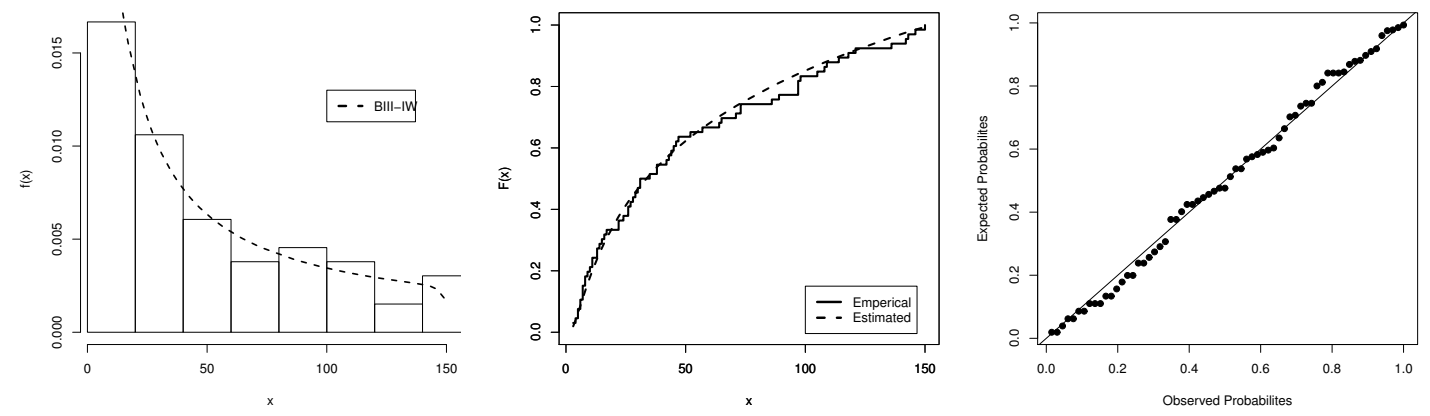

Figure 6: Fitted pdf (left), cdf (center) and P-P(right) plots of the BIII-IW distribution for daily new COVID-19 confirmed cases in China

\section{Conclusion}

We proposed a new probability distribution, called BIII-IW distribution, based on inverse Weibull and Burr III distribution via T-X family method. Its pdf and hrf shapes have very flexible forms. We studied certain mathematical properties such as random number generator, sub-models, ordinary moments, conditional moments, residual life functions, reliability measures and characterizations. We addressed the maximum likelihood estimation for the BIII-IW parameters and evaluated the precision of the maximum likelihood estimators via a simulation study. We considered applications to daily new COVID-19 confirmed cases in Pakistan and daily new COVID-19 deaths in China. We computed various model selection criteria for the BIII-IW distribution. The BIII-IW distribution is flexible, competitive and parsimonious with potential for wide ranging applications. 


\section{References}

Abbas, S., Hameed, M., Cakmakyapan, S., and Malik, S. (2020), On gamma inverse Weibull distribution. Journal of the National Science Foundation of Sri Lanka, 47(4), 445-453.

Abbas, S., Taqi, S., Mustafa, F., Murtaza, M., and Shahbaz, M. (2017), Topp-Leone Inverse Weibull Distribution: Theory and Application.European Journal of Pure and Applied Mathematics, 10(5), 1005-1022.

Alzaatreh, A., Mansoory, M., Tahirz, M. H., Zubair, M., and Ghazalik, S. A. (2016), The gamma half-Cauchy distribution: Properties and applications. Hacettepe Journal of Mathematics and Statistics, 45(4), 1143-1159.

Alzaatreh, A., Lee, C., and Famoye, F. (2013), A new method for generating families of continuous distributions. Metron, 71(1), 63-79.

Bantan, R. A., Chesneau, C., Jamal, F., and Elgarhy, M. (2020), On the Analysis of New COVID-19 Cases in Pakistan Using an Exponentiated Version of the M Family of Distributions. Mathematics, 8(6), 953.

Bhattacharyya, G. K., and Johnson, R. A. (1974), Estimation of reliability in a multicomponent stress-strength model. Journal of the American Statistical Association, 69(348), 966-970.

Elbatal, I., Condino, F., and Domma, F. (2016), Reflected generalized beta inverse Weibull distribution: definition and properties. Sankhya B, 78(2), 316-340.

Eliwa, M. S., El-Morshedy, M., and Ali, S. (2020), Exponentiated odd Chen-G family of distributions: statistical properties, Bayesian and non-Bayesian estimation with applications. Journal of Applied Statistics, 1-27.

Fayomi, A. (2019). The odd Frechet inverse Weibull distribution with application. Journal of Nonlinear Sciences and Applications, 12, 165-172.

Glänzel W. A. (1987). Characterization theorem based on truncated moments and its application to some distribution families. Mathematical Statistics and Probability Theory (Bad Tatzmannsdorf, 1986), Vol. B, Reidel, Dordrecht, 75-84.

Hafida G. and Haitham Y. H. (2019), Validation of Burr XII inverse Rayleigh model via a modified chi-squared goodness-of-fit test. Journal of Applied Statistics, 47(3), 393-423. 
Khan, M. S. (2010), The beta inverse Weibull distribution. International Transactions in Mathematical Sciences and Computer, 3(1), 113-119.

Khan, M. S., and King, R. (2016), New generalized inverse Weibull distribution for lifetime modeling. Communications for Statistical Applications and Methods, 23(2), 147161.

Keller, A.Z. and Kamath, A.R.R. (1982), Alternative Reliability Models for Mechanical Systems. Proceeding of the 3rd International Conference on Reliability and Maintainability, 411-415.

Kotz S., Lai CD and Xie M. (2003), On the Effect of Redundancy for Systems with Dependent Components. IIE Transactions, 35(12), 1103-1110.

Eliwa M. S. , El-Morshedy M. and Ali S., (2020), Exponentiated odd Chen-G family of distributions: statistical properties, Bayesian and non-Bayesian estimation with applications. Journal of Applied Statistics. https://doi.org/10.1080/02664763.2020.1783520

Shahbaz, M. Q., Shahbaz, S., and Butt, N. S. (2012), The Kumaraswamy-Inverse Weibull Distribution. Pakistan Journal of Statistics and Operation Research, 8(3), 479-489. 